19 Revue d'histoire du XIXe siècle

Société d'histoire de la révolution de 1848 et des

révolutions du XIXe siècle

$54 \mid 2017$

La part animale du XIX ${ }^{e}$ siècle

\title{
À la redécouverte du capitalisme américain
}

Nicolas Barreyre et Alexia Blin

\section{OpenEdition}

\section{Journals}

Édition électronique

URL : http://journals.openedition.org/rh19/5194

DOI : $10.4000 /$ rh 19.5194

ISSN : $1777-5329$

Éditeur

La Société de 1848

Édition imprimée

Date de publication : 1 août 2017

Pagination : 135-148

ISSN : 1265-1354

Référence électronique

Nicolas Barreyre et Alexia Blin, «À la redécouverte du capitalisme américain », Revue d'histoire du XIXe siècle [En ligne], 54 | 2017, mis en ligne le 01 juillet 2019, consulté le 02 janvier 2020. URL : http:// journals.openedition.org/rh19/5194; DOI : 10.4000/rh19.5194

Ce document a été généré automatiquement le 2 janvier 2020.

Tous droits réservés 


\title{
À la redécouverte du capitalisme américain
}

\author{
Nicolas Barreyre et Alexia Blin
}

1 «Un spectre hante les départements d'histoire des universités », annonce le New York Times en 2013. Ce spectre, c'est celui du capitalisme. Pendant des décennies, les historiens auraient délaissé ce phénomène majeur, tout occupés qu'ils étaient à recouvrer l'expérience et la capacité d'action des sans-voix et réécrire l'histoire « par le bas ». Mais une nouvelle génération s'attellerait désormais à analyser comment s'est construit le capitalisme américain. Une tâche que la crise financière de 2008 a soudain rendu urgente, et visible ${ }^{1}$.

2 Cette «nouvelle histoire du capitalisme » rencontre depuis quelques années un succès médiatique remarqué, dont l'article du New York Times a été à la fois le signe et le moteur. Plusieurs ouvrages ont connu une couverture de presse extensive (fait rare pour les ouvrages universitaires), renforcée par l'extraordinaire succès américain du Capital au XXI ${ }^{e}$ siècle de Thomas Piketty ${ }^{2}$. Ce regain d'intérêt historiographique se traduit par une activité éditoriale importante, ainsi que par une place institutionnelle grandissante accordée aux questions économiques dans les départements d'histoire américains, contribuant ainsi à renforcer leur visibilité.

3 S'il ne faut pas exagérer l'importance quantitative du phénomène, il est certain que la "nouvelle histoire du capitalisme» (NHC) a acquis une centralité au sein du débat historiographique qu'il aurait été bien difficile de prédire il y a dix ans. Qu'est-ce donc que cet objet historiographique mal identifié ? Ni simple sous-champ orienté sur un objet commun, ni tournant épistémologique, la NHC résulte principalement d'une convergence de travaux qui se reconnaissent, plus ou moins, sous un label commun, marquage institutionnel et historiographique rassemblant des démarches intellectuelles labiles, souvent individuelles, mais congruentes. Ce sont les mécanismes et les effets de ce mouvement que cet essai propose de décrire et d'évaluer. 


\section{Une opération de mobilisation historiographique}

4 Qu'est-ce que la nouvelle histoire du capitalisme? Après tout, ce n'est pas comme si l'économie avait complètement disparu des départements d'histoire, même si elle y a été repoussée dans les marges. Si en 2005 seuls $2 \%$ des postes étaient officiellement profilés sur de l'histoire économique, ce chiffre sous-estime le nombre d'historiens abordant des objets économiques ${ }^{3}$. Ce qui caractérise ces travaux, toutefois, c'est leur éparpillement, et leur division en niches historiographiques qui communiquent peu: histoire économique (au sens étroit du terme), histoire des entreprises (business history), histoire ouvrière (labor history), ou histoire de la consommation. Certaines de ces niches sont d'ailleurs majoritairement explorées dans d'autres départements que ceux d'histoire, ce qui accroît leur invisibilité dans une grande partie de la profession ${ }^{4}$.

5 Dans ce contexte, la NHC est d'abord un label - ce mot a son importance - qui permet la coalescence de travaux individuels qui cherchent à faire sens de l'expérience contemporaine du capitalisme aux États-Unis. Dans la veine présentiste assumée de l'historiographie américaine, beaucoup des travaux qui se revendiquent de cette histoire partagent cette volonté d'expliquer le capitalisme comme un type de rapport social, sans qu'il s'agisse là d'une référence théorique à la pensée marxiste. Dans les dix dernières années, des historiens ont ainsi exploré, comme un rapport social et politique (lui-même générateur d'institutions), la relation des Américains au risque économique, au succès ou à l'échec professionnel, au crédit et à la dette, à la bourse et aux marchés financiers, à la valeur et à l'argent ${ }^{5}$. Ces ouvrages, au-delà de leurs qualités scientifiques, partagent l'évidence que la société américaine est capitaliste, jusqu’à parfois postuler une équivalence entre les deux termes, comme en témoignent certains titres, qui semblent présenter divers aspects du capitalisme comme des caractéristiques intrinsèques de la nation étasunienne ${ }^{6}$. Ils ont par ailleurs en commun de considérer que cette omniprésence du capitalisme crée un certain nombre de problèmes politiques qu'il faut éclairer économiquement.

6 De tels questionnements ont aidé à rendre visible un ensemble de livres individuels pourtant épars. La crise des subprimes de 2008 ayant rendu urgent, dans le débat public, ce type d'interrogations, les historiens du capitalisme, groupe peu nombreux et éclectique, se sont retrouvés les seuls de leur profession à offrir un regard critique centré sur ce système économique - dont les dysfonctionnements graves deviennent soudain évidents - alors que la majorité des économistes paraissent, eux, les grands prêtres de cette religion déchue. Quoique le fruit de travaux entamés pour la plupart avant le déclenchement de la crise, les ouvrages des nouveaux historiens du capitalisme sont pour beaucoup publiés après 2008, et leurs auteurs peuvent insister sur l'actualité brûlante des sujets dont ils traitent. Se présentant comme explicitement critiques, ils revendiquent une place dans le débat public sur la montée des inégalités et les excès du système économique ${ }^{7}$.

7 Mais le contexte politique coïncide aussi avec un moment historiographique particulier, avec le sentiment qui se répand d'avoir atteint les limites du tournant culturaliste ${ }^{8}$ des années 1980 . Une série d'initiatives et de manifestes par des historiens en vue appelle ouvertement à sortir du paradigme dominant pour se tourner vers diverses questions et méthodes jugées désormais plus importantes, et plus en phase avec la réalité du monde en-dehors de la tour d'ivoire universitaire. Ces prises de position, souvent polémiques, ne font pas l'unanimité, et provoquent le plus souvent 
des controverses virulentes guère exemptes de caricatures. Mais ces débats révèlent aussi une profession historienne qui se cherche, ou plutôt qui cherche confusément une sortie à une impasse où se mêlent sentiment de marginalisation dans le discours public et remises en cause historiographiques'.

8 Il ne fait guère de doute que, au moins pour ses promoteurs les plus en vue, la NHC est aussi une arme pour faire bouger les lignes et questionnements historiographiques. La proposition toutefois n'est pas celle d'une innovation épistémologique, un de ces nombreux «turns » qui ont émaillé les débats historiographiques depuis les années 1980 et ont substitué l'impératif d'originalité à la cumulativité dans la science historique ${ }^{10}$. Il s'agit au contraire de faire d'un objet le centre des recherches; de faire du capitalisme lui-même un phénomène à investiguer directement. Bref, ce n'est pas un tournant épistémologique mais une opération historiographique de mobilisation.

Dans cette perspective, les historiens du capitalisme font preuve d'un réel effort prosélyte, à destination de leurs collègues, des étudiants, mais aussi du grand public. De nouvelles collections sont lancées chez des éditeurs universitaires. Des dossiers spéciaux paraissent dans les grandes revues ${ }^{11}$. D'autres initiatives fleurissent pour nourrir l'attractivité et la visibilité du champ, souvent appuyées sur un petit nombre d'institutions puissantes comme Harvard ou Cornell. Il existe désormais une école d'été, un cours en ligne et un podcast, par exemple, pour présenter les travaux des jeunes historiens du capitalisme sur la Réserve fédérale, la lutte des hommes d'affaires contre le New Deal, l'industrie pharmaceutique ou la discrimination raciale ${ }^{12}$.

Cependant, la NHC ne s'est pas constituée en école mais bien en label. Les textes, forums et manifestes sur l'histoire du capitalisme ont en commun un grand éclectisme d'approches et d'objets ${ }^{13}$. Dans un passage en revue historiographique datant de 2011, Sven Beckert assume largement ce côté éparpillé, revendiquant même pour ce nouveau champ des ouvrages qui ne s'en réclamaient pas. Une table ronde dans une revue américaine centrale à la discipline témoigne de la même absence de cohérence ${ }^{14}$. Les conférences organisées récemment insistent sur le pluriel des approches et des objets, et même des capitalismes ${ }^{15}$. En cela, la NHC n'est ni une école ni une démarche intellectuelle concertée. Mais elle permet de donner une force d'ensemble à des travaux qui se trouveraient autrement isolés. Elle donne le poids du groupe, la dynamique d'un mouvement collectif, à des sous-champs séparés. Elle fait dialoguer une histoire du travail qui a perdu sa centralité d'antan avec l'histoire politique, la business history, et la part de l'histoire sociale et culturelle qui s'intéresse à la dimension économique de la vie des Américains.

11 En ce sens, si la NHC n'a pas réinventé un intérêt historien pour l'économique, elle lui a incontestablement insufflé une dynamique en offrant un lieu partagé, et en vue, à des travaux épars, ou en permettant de redécouvrir la dimension économique, jusque-là sous-jacente, de nombreux ouvrages. On peut citer ici le cas du livre de William Cronon, Nature's Metropolis, qui porte sur la manière dont les activités industrielles et commerciales de Chicago ont remodelé la géographie, et la nature-même, des Grandes Plaines au XIX ${ }^{\mathrm{e}}$ siècle. Paru en 1991, l'ouvrage devient d'abord un classique de l'histoire de l'environnement. Les effets de la mobilisation du «capital» tiennent une grande place dans l'analyse, mais le terme de "capitalisme " n'apparaît que très peu, et ne donne pas lieu à des développements particuliers. Le livre a pourtant fait l'objet d'une réappropriation récente de la part des historiens du capitalisme, qui l'utilisent dans 
leurs programmes de cours, en insistant sur les problématiques proprement économiques de l'ouvrage ${ }^{16}$.

Ce type d'opération est indiscutablement une réussite historiographique, qui se mesure à la visibilité d'ensemble de ce groupe de travaux, et la recrudescence du terme " capitalisme » dans les titres de livres d'histoire américaine ${ }^{17}$. Elle s'observe également dans la multiplication des cours portant explicitement sur l'histoire du capitalisme dans les universités, ainsi que des postes spécifiquement dédiés qui commencent à $s^{\prime}$ ouvrir $^{18}$. Il serait faux toutefois de ne voir dans cette entreprise qu'un effet de marketing universitaire, indéniable malgré tout et qui correspond bien aux structures professionnelles de la recherche étatsunienne ${ }^{19}$. Il s'agit également d'une offensive historiographique pour redonner une place aux historiens, et à l'histoire comme discipline, dans l'étude de l'économie. Par construction, l'entreprise n'est pas pluridisciplinaire. L'idée est à la fois d'encourager les historiens à déplacer leurs objets de prédilection pour revenir à des problématiques économiques, et d'arracher aux économistes un quasi-monopole d'autorité dans ce domaine ${ }^{20}$.

13 Cette opération ne peut se comprendre que dans le contexte d'une grande divergence amorcée dans les années 1970 entre les économistes-historiens (economic historians), d'une part, et les historiens de l'autre. La conversion massive des départements d'économie américains à l'économétrie, à l'abstraction mathématisée du monde reposant sur des postulats épistémologiques étroits - ce qu'on désigne aujourd'hui du nom « d'économie orthodoxe » en France - a entraîné dans son sillage les praticiens de l'histoire économique jusqu'à rendre incompréhensibles aux historiens non initiés les travaux économistes sur le passé américain ${ }^{21}$. Cette pente "cliométrique " a coïncidé avec un mouvement inverse dans les départements d'histoire, vers une histoire sociale très culturaliste travaillant des questions très lointaines de celle de l'histoire économique contemporaine. La longue absence de dialogue qui en a résulté ouvre aujourd'hui un espace à une initiative historienne comme celle de la NHC, qui prend les outils des historiens pour ostensiblement réinvestir un champ économique labouré dans une perspective très spécifique par des économistes-historiens eux-mêmes dominés, voire marginalisés, dans les départements d'économie ${ }^{22}$.

\section{La bataille sur capitalisme et esclavage}

Le débat actuel où cette divergence est la plus marquante est celui des liens entre esclavage et capitalisme aux États-Unis. La NHC ne s'est pas uniquement concentrée sur cette question, mais la sortie rapprochée de plusieurs ouvrages, dont certains offrent le flanc à la polémique, en fait un point nodal mais complexe du contentieux, et il est utile de s'attarder dessus.

Voilà une dizaine d'années que des historiens ont réinvesti la question du rapport entre capitalisme et esclavage en histoire américaine. Cet intérêt n'est pas nouveau, mais ces ouvrages récents renversent la perspective qui prévalait jusqu'alors. Les débats des années 1970, qui comprenaient le capitalisme comme un rapport de production fondé sur la dissociation entre le travailleur et sa force de travail, avaient généralement conclu que l'esclavage était une institution pré-capitaliste, et son existence expliquait en grande partie ce qui était conçu comme l'arriération économique durable du Sud des États-Unis ${ }^{23}$. Les travaux récents, eux, s'attachent au contraire à mettre l'esclavage au cœur du capitalisme, soit mondial, soit plus sûrement américain. Cette centralité est 
défendue de deux manières. L'une consiste à insister sur l'importance de la production du coton dans l'économie globale américaine, aussi bien pour les revenus qu'elle génère directement et indirectement que pour la dépendance de la première industrialisation, essentiellement dans le Nord-Est du pays, sur la production et les commandes des grandes plantations sudistes. Aussi le décollage industriel, et donc le capitalisme américain, reposait essentiellement sur un coton dont la culture en quantités adéquates était impossible sans recours à une main-d'œuvre servile. Le second argument est intrinsèque au système esclavagiste sudiste au XIX ${ }^{e}$ siècle. Il consiste à dire que la grande plantation a recours à tous les outils de l'entreprise « moderne ", notamment la comptabilité, et qu'elle poursuit ce but capitaliste par excellence: une productivité toujours croissante pour maximiser les profits ${ }^{24}$.

16 Il s'agit là d'une perspective qui se veut critique. Les anciens travaux avaient, disent ces auteurs récents, absous le capitalisme de l'esclavage parce que celui-ci était un système d'un autre âge, que le capitalisme triomphant avait relégué, par la guerre de Sécession, au rebut de l'histoire. Au contraire, selon eux, l'esclavage était bien au cœur d'un système qui n'aurait pu décoller sans lui. C'est cette consubstantialité qui explique que l'esclavage soit toujours aujourd'hui un problème politique aux États-Unis. C'est le péché originel du capitalisme (américain). Et c'est, dans le contexte politique de Black Lives Matter, autant une condamnation du capitalisme que de l'esclavage et ses séquelles actuelles ${ }^{25}$.

17 Les débats vifs provoqués par cet ensemble de publications récentes sont intéressants pour ce qu'ils révèlent des lignes de fracture disciplinaires et historiographiques. La divergence la plus forte reste avec les économistes-historiens. Une partie des objections portent sur les mesures: il est vrai que certains travaux sur esclavage et capitalisme manient parfois des chiffres à tort et à travers, et si cela ne remet pas toujours en cause leur thèse cela les dessert forcément ${ }^{26}$. Mais ce type de critique ne sort pas de l'ordinaire de l'évaluation par les pairs. Il est toutefois une série d'objections plus fondamentales. Les économistes-historiens qui ont recensé ces travaux récents se sont particulièrement élevés contre la thèse de la centralité de l'esclavage. Pour eux en effet, une telle affirmation implique une proposition contrefactuelle : sans l'esclavage, il n'y aurait pas eu de capitalisme. Cela va dans la droite ligne de la méthode économique, censée établir des relations causales strictes dans un système d'équilibre général. Une cause ne peut être cause que si elle est nécessaire. Les historiens répliquent qu'ils ont mis en évidence le rôle crucial de la production esclavagiste de coton dans l'essor du capitalisme américain ou mondial. Pour eux, la question n'est pas tant de savoir si un capitalisme aurait pu, in abstracto, apparaitre sans esclavage, mais que le capitalisme historique a de fait suivi une trajectoire dépendante de ce système - et c'est cela qu'il faut expliquer. D'un côté fleurit l'accusation, un rien scientiste, d'absence de « rigueur social-scientifique»; de l'autre vient la réponse que les préoccupations des économistes-historiens les empêchent tout simplement d'expliquer un phénomène historique pourtant massif, dont la dimension économique ne peut être ignorée ${ }^{27}$.

18 Il serait faux, toutefois, de résumer le débat sur esclavage et capitalisme à un face-àface entre historiens et économistes-historiens. Parmi les historiens, s'élèvent en effet des critiques qui dépassent l'ordinaire des recensions d'ouvrage pour porter sur l'entreprise-même de la NHC. L'une des plus récurrentes est l'accusation de revenir à une histoire des dominants, faite uniquement par des hommes blancs puissants, tout en effaçant à nouveau ce que la nouvelle histoire sociale a mis quarante ans à explorer : la 
capacité d'action des dominés, cette agency au cœur de toute cette historiographie ${ }^{28}$. L'accusation est parfois incantatoire, de l'ordre du réflexe. On est là dans la dimension politique de l'épistémologie historienne ${ }^{29}$. On aurait tort de ne pas la prendre au sérieux, d'abord parce qu'elle a des effets forts sur le type d'histoire qui s'écrit. Certains ouvrages d'ailleurs tentent d'intégrer le rôle et la place des esclaves dans cette histoire d'oppression capitaliste, avec parfois des effets de ventriloquisme malheureux. On voit toutefois ici que la démarche critique devenue la marque de la discipline historique ne suffit pas à la réception des travaux de la NHC par les historiens: pour certains, l'approche adoptée par la NHC crée un problème de légitimitée ${ }^{30}$.

\section{Dénaturaliser le capitalisme}

19 Dans sa configuration actuelle, la NHC fait polémique. C'est en partie parce qu'elle agace par une visibilité médiatique qui est aussi un effet marketing. C'est le résultat, également, de limites réelles à ce champ tel qu'il fonctionne actuellement, et sur lesquelles nous allons revenir. Mais il importe auparavant d'expliciter les effets de ce regroupement relativement récent sur l'histoire américaine - puisque la NHC, quoi qu'elle ait une visée d'histoire globale, occupe principalement sous son label des américanistes $^{31}$.

$\mathrm{Au}$ fond, ce qui caractérise la NHC, c'est de s'intéresser à la dimension économique de l'histoire américaine sans être en soi une histoire économique. À nouveau, il faut faire la part de l'hétérogénéité des travaux, mais ceux qu'on reconnaît le plus facilement sous le label NHC font plus une histoire culturelle et sociale des acteurs économiques qu'une histoire proprement économique. En cela la NHC est pleinement héritière du tournant critique des années 1980, en ce sens qu'elle ne peut pas concevoir contrairement à l'histoire économique actuellement dominante aux États-Unis d'économie qui fonctionne $\grave{a}$ part $^{32}$. Les économistes-historiens y reconnaissent l'influence de Karl Polanyi ${ }^{33}$ (parfois revendiquée, parfois absente des travaux de la $\mathrm{NHC}$ ). Mais c'est plus généralement l'aboutissement logique du déconstructivisme des trente dernières années. En cela, la NHC est moins rebelle qu'elle ne le semble de prime abord.

21 Il faut insister là-dessus. Ce que travaille la NHC, au fond, c'est à quel point rien n'est naturel dans les relations économiques, tout est construit historiquement. Le capitalisme américain est un processus "contingent», "contesté », "qui émerge et prend forme à travers « les menus détails confus de la vie quotidienne» » ${ }^{34}$. Le rapport à l'argent, aux institutions financières, et la confiance dans le "marché » ne sont pas le résultat heureux de "bonnes » institutions économiques, mais au contraire de relations sociales et politiques, de propagande et de conflits d'intérêts précisément situés. L'émergence de la figure de l'entrepreneur n'est pas le résultat d'institutions politiques et légales «libérant» les énergies, mais l'aboutissement complexe et heurté de transformations des notions de risque, de succès et d'échec, et leur incorporation dans le fonctionnement social ordinaire. La mobilisation de viviers de main-d'œuvre n'est pas la rencontre, optimale ou biaisée, entre l'offre et la demande sur le marché du travail, mais les effets situés d'engagements sociaux, politiques et religieux où la dimension économique n'en est qu'une parmi d'autres ${ }^{35}$.

La NHC a donc l'immense avantage d'explorer les dimensions culturelles, sociales (et plus rarement politiques) des relations économiques sans jamais postuler qu'on peut 
les observer en-dehors de ces encastrements. Ce faisant, elle a déplacé un certain nombre de débats historiens antérieurs. Le premier posait la question des origines du capitalisme américain. Il s'agissait de déterminer quand il était apparu et quand il était devenu dominant en Amérique du Nord. Certains le faisaient remonter au XVII siècle des marchands puritains. D'autres voyaient l'époque de la révolution américaine comme un tournant majeur, alors que d'autres encore, prolongeant une historiographie plus ancienne, voyaient la guerre de Sécession comme le moment du triomphe du capitalisme. Ce débat était lié à un second, qui tournait autour de la thèse que les ÉtatsUnis auraient connu dans les premières décennies du XIX siècle une "révolution du marché ", qui aurait inexorablement piégé les Américains, notamment les fermiers, dans les rets d'un marché de plus en plus englobant et impersonnel, les forçant à vendre leur production sur de très longues distances et à des acheteurs anonymes, et subissant ses fluctuations et diktats. Cette transformation drastique a été beaucoup débattue et contestée - quoique les désaccords portassent surtout sur le degré d'adhésion volontaire par les acteurs à de telles transformations ${ }^{36}$. Elle est restée longtemps au cœur de l'histoire de cette période. Elle partageait avec le débat sur les origines du capitalisme l'idée qu'il s'agissait d'un système fait de forces mues par les humains, certes, mais pourtant impersonnelles.

Ces débats ont largement disparu avec les années 1990. La NHC, par la suite, les a déplacés, passant ainsi de l'étude du marché à celle de la marchandisation (commodification), opération sociale et culturelle reconceptualisant un objet pour en faire le lieu d'une valeur comptable et donc, potentiellement, d'un échange marchand. De nombreux travaux critiques ont insisté sur ce phénomène, qui permet par exemple de redéfinir l'esclavage comme une entreprise foncièrement capitaliste justement parce qu'elle marchandise le travailleur lui-même. Cette focale s'accompagne d'une grande attention aux signes et techniques qui font la valeur: les opérations d'évaluation, de dénombrement, de transferts, et d'investissement symbolique que fait le livre de compte ${ }^{37}$. Elle explique aussi pourquoi la NHC marque un tournant vers la dimension financière du capitalisme - «les baguettes magiques mercantiles de la spéculation, du crédit, et des mouvements globaux des prix»-, s'éloignant ainsi des questions d'industrialisation et de rapports de production qui préoccupaient les générations antérieures ${ }^{38}$. Le capitalisme ici n'est plus objectivé mais culturalisé. Il est "plus un climat qu'un ordre social» ${ }^{39}$. Et il s'agit là d'un déplacement clé. La NHC s'intéresse peu, jusqu'à présent, aux origines du capitalisme, dans le sens où elle ne s'interroge guère sur ce qui est hors capitalisme. Elle se pose au contraire au milieu du capitalisme et analyse (et historicise) ses dimensions socio-culturelles, et politiques dans le sens où la relation capitaliste est une relation de pouvoir encodée dans la société américaine ${ }^{40}$.

\section{Le refus revendiqué de la définition et ses limites}

Pour féconde qu'elle soit parfois, cette approche n'en a pas moins ses limites. La plus centrale est sans doute l'absence de définition de ce que les auteurs de la NHC entendent par "capitalisme ». C'est probablement le reproche le plus récurrent dans les débats actuels (même si, pour être juste, rares sont ces critiques qui proposent euxmêmes des définitions de travail ${ }^{41}$ ). Il s'agit là pourtant d'une posture revendiquée par certains apôtres de la NHC. Seth Rockman, dans un essai historiographique? assume « 
ne s'investir que minimalement dans la recherche d'une définition fixe ou théorique du capitalisme ». Louis Hyman affirme ailleurs que « le problème avec le capitalisme n'est pas de le définir, mais de comprendre pourquoi il résiste aux définitions simples. [...] Définir simplement le capitalisme est une mauvaise idée $»^{42}$. Pour eux, il s'agit de ne pas se laisser embourber dans des querelles théoriques et perdre ainsi de vue l'étude du capitalisme in concreto, comme cela serait arrivé par le passé. Ce faisant, ils refusent activement de débattre avec les travaux plus anciens, en particulier d'inspiration marxiste, qui ont traité des mêmes questions ${ }^{43}$. Au contraire, affirment-ils, il faut explorer les histoires du capitalisme - pluriel - dans tous ses avatars, ses variétés si l'on

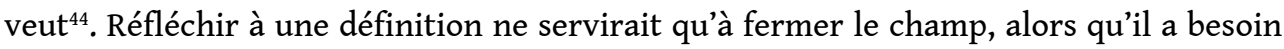
au contraire de s'ouvrir à toutes les perspectives pour renouveler les approches.

Il y a là une posture qui permet de revendiquer une gamme très large de travaux de toutes sortes pour la NHC, tout en laissant chaque historienne et historien, individuellement, se réclamer d'un courant historiographique qui a désormais le vent en poupe. Il n'en reste pas moins qu'à perdurer ce refus empêche le champ de trouver sa cohérence intellectuelle, voire de nous aider à faire sens du capitalisme, alors qu'il est ostensiblement l'objet à expliquer, ou à tout le moins à historiciser. Déjà le débat sur les origines du capitalisme avait souffert d'une définition trop floue, se réduisant souvent au "marché ", ce qui permettait chacun de voir un tournant à sa porte ${ }^{45}$. Les historiens de la NHC, même dans les rares cas où ils consentent à formuler une définition? comme lorsqu'ils insistent sur la marchandisation au cœur de leurs histoires, reproduisent la confusion entre économie de marché et capitalisme. Mais plus encore que les travaux précédents, le déplacement de focale et l'absence de définition qui caractérisent la NHC semblent impliquer que tout peut entrer dans le capitalisme. Les petites ventes de rue et les stratégies de survie dans les villes américaines du premier XIX siècle sont soudain du capitalisme entrepreneurial ${ }^{46}$. Le mot devient particulièrement englobant, et tout entre dans son giron ${ }^{47}$. Le débat sur l'esclavage, par exemple, est en partie le symptôme de cette absence de définition autant que d'une véritable reconceptualisation.

Il y a, en filigrane, un risque évident à entretenir le flou : une sorte d'exceptionnalisme sourd, larvé. La plupart des auteurs de la NHC font comme s'il allait de soi que l'économie américaine était capitaliste, au moins depuis la Révolution si ce n'est avant, et dès lors que toute relation économique était capitaliste. On suppose que, pressés de préciser leur pensée, ils récuseraient cette caractérisation. Mais l'absence même de discussion sur la définition, ou tout au moins sur le périmètre du capitalisme, revient à y englober historiquement toutes les transactions économiques. Certains ont préconisé d'autres appellations, pour mettre fin à l'ambiguïté de ce qu'étudie réellement la NHC. Michael Zakim, par exemple, suggère dans un essai critique d'utiliser le label « histoire de l'économie » (history of the economy), soulignant qu'il serait " plus difficile d'en faire un cliché, mais qu'il mettrait effectivement le capitalisme à sa place, au sein d'une histoire plus large de propriété, de privilège, de pouvoir, d'autorité, d'ordre, et d'(in)stabilité ». Richard John, dans un texte antérieur à la médiatisation de la NHC, promouvait le retour à la notion d'économie politique, en ce qu'elle porte en elle une conception encastrée des relations économiques ${ }^{48}$. Il faut avouer, toutefois, que ces termes n'ont pas la force, en terme de marketing historiographique, de la «nouvelle histoire du capitalisme». Or dans l'économie universitaire américaine, faite de 
concurrence entre cours pour attirer les étudiants et entre manuscrits pour l'attention éditoriale, c'est là un facteur non négligeable pour construire un champ et le peupler.

En l'état actuel, la NHC a donc acheté son dynamisme et sa visibilité au prix de la rigueur intellectuelle non pas de ses approches - contrairement à l'accusation de certains économistes-historiens - mais de sa (non-)conceptualisation de la catégorie même de capitalisme. Dans la pratique de la plupart de ces auteurs, le capitalisme est en réalité une notion culturelle plus qu'un système économique ${ }^{49}$. C'est ici, au fond, la force de la proposition : elle en fait un objet historique malléable et historicisable. C'est aussi sa faiblesse, en un sens, car les dimensions structurelles et institutionnelles de l'économie sont souvent peu présentes ou peu pensées dans ces études, malgré les exceptions. En refusant d'affronter la question de la définition, ne serait-ce qu'à petits pas mais collectivement, la NHC abdique en fait (ou à tout le moins repousse à plus tard) l'idée même de comprendre ce qu'est le capitalisme comme système pour n'en faire que le synonyme d'une économie où les transactions médiatisées par un " marché » sont centrales. Ironiquement, cela les rend fort proches des économistes(historiens) qu'ils critiquent pourtant frontalement.

La bonne nouvelle, c'est que les dimensions économiques de l'histoire américaine (et globale) sont revenues au centre des préoccupations des historiens, ou plutôt d'un groupe encore petit mais grandissant et attractif parmi eux. L'économie redevient un enjeu historique visible, non en soi mais immergé dans ses relations sociales, culturelles, et politiques. Pas de «désencastrement» ici, bien au contraire. Cette " nouvelle histoire " n'est sans doute pas partie pour expliquer ce qu'est le capitalisme, malgré le slogan. Elle promet malgré tout d'explorer comment il a fonctionné et s'est transformé historiquement dans le contexte américain, de l'intérieur pour ainsi dire. Et il $\mathrm{y}$ a là de nombreux chantiers passionnants à mener.

\section{NOTES}

1. Jennifer Schuessler, 'In History Departments, It's Up With Capitalism', New York Times, 6 avril 2013.

2. Thomas Piketty, Le capital au XXI siècle, Paris, Éditions du Seuil, 2013 ; traduction américaine par Arthur Goldhammer, Cambridge (Mass.), Harvard University Press, 2014. Parmi les livres ayant reçu une grande couverture médiatique, on peut citer Sven Beckert, Empire of Cotton: A Global History, New York, Alfred A. Knopf, 2014, désigné par le New York Times comme l'un des dix meilleurs livres de l'année 2015: 'The 10 Best Books of 2015', New York Times, 3 décembre 2015.

3. Robert B. Townsend, 'What's in a Label? Changing Patterns of Faculty Specialization since 1975', Perspectives on History, January 2007.

4. Il en est ainsi de la business history, surtout faite dans les business schools des universités, ainsi que de l'histoire économique désormais presque exclusivement menée dans les départements d'économie.

5. Jonathan Levy, Freaks of Fortune: The Emerging World of Capitalism and Risk in America, Cambridge (Mass.), Harvard University Press, 2012; Scott A. Sandage, Born Losers: A History of Failure in America, Cambridge (Mass.), Harvard University Press, 2005 ; Louis Hyman, Debtor Nation: The 